\title{
Taugt die aggregierte Delinquenzrate einer Schulklasse als Maßzahl für die Bestimmung kriminogener Peer-Effekte?
}

\section{Is the Aggregated Delinquency Rate of a School Class a Suitable Indicator for Determining Criminogenic Peer Effects?}

\author{
Ausgewählte Probleme und Lösungsmöglichkeiten \\ Selected Problems and Solutions
}

https://doi.org/10.1515/mks-2020-2039

Zusammenfassung: In der kriminologischen Forschung werden Effekte delinquenter Peerexposition auf das Legalverhalten junger Menschen gerne im Bezugsrahmen statistischer Mehrebenenanalysen bestimmt, in welchen die auf Kontextebene hochaggregierten Selbstauskünfte der befragten Personen als Indikator für die Kriminalitätsbelastung der Freunde verwendet werden. Gerade im europäischen Raum liegen solchen Untersuchungen häufig schulbasierte Stichproben zugrunde. Stillschweigend angenommen wird dabei, dass sich erstens die Freunde eines Jugendlichen aus den Schulkameraden rekrutieren und dass zweitens die aggregierte Delinquenzrate einer Schulkasse eine geeignete Messgröße für die Berechnung des Peer-Effektes darstellt. Dieser Beitrag problematisiert die zweite Annahme und weist nach, dass einerseits das Abstellen auf die unkorrigierte Klassenkriminalitätsrate artifizielle Befunde produziert, weil es den einzelnen Jugendlichen in die Bildung der Expositionsvariablen miteinbezieht (Ego-Bias-Problem), und andererseits der Rückgriff auf herkömmliche Mehrebenenanalysen die reziproken Wirkungsdynamiken zwischen jungen Menschen und ihren Klassenkameraden (Simultanitätspro-

\footnotetext{
*Kontaktperson: Assoz. Univ.-Prof. Dr. Helmut Hirtenlehner, Zentrum für Kriminologie, Johannes Kepler Universität Linz, Altenber-

gerstr. 69, 4040 Linz, Österreich, E-Mail: helmut.hirtenlehner@jku.at Univ.-Prof. Dr. Johann Bacher, Institut für Soziologie, Johannes Kepler Universität Linz, Altenberger Straße 69, 4040 Linz, Österreich, E-Mail: johann.bacher@jku.at

Dr. Christoph Weber, Pädagogische Hochschule Oberösterreich, Kaplanhofstraße 40, 4020 Linz, Österreich,

E-Mail: christoph.weber@ph-ooe.at
}

blem) vernachlässigt. Beide Umstände können auf eine Überschätzung kriminogener Peer-Effekte hinauslaufen. Im gegenständlichen Beitrag werden Modellierungsvarianten, die hier geeignete Abhilfe versprechen, vorgestellt und beispielhaft auf die Ladendiebstahlsdelinquenz von Kindern und Jugendlichen angewandt. Sowohl Instrumentalvariablenregressionsanalysen als auch nicht-rekursive Strukturgleichungsmodelle erbringen Belege für eine moderate Abhängigkeit der Ladendiebstahlsprävalenz adoleszenter Personen vom Umfang der Diebstahlskriminalität ihrer Klassenkameraden.

Schlüsselwörter: Delinquente Peers, Instrumentalvariablenanalyse, Ladendiebstahlskriminalität

Abstract: In criminological research, criminogenic peer effects are sometimes estimated using multilevel models for school class based self-report data, whereby the aggregated crime prevalence rate of a class is employed as contextual-level predictor of an individual's crime involvement. This approach assumes that, first, young people recruit their friends from the pool of their classmates, and second, that the aggregated delinquency rate of a school class is a proper indicator for estimating the delinquent peer effect. This article examines the second assumption and shows that the approach described above leads to biased results. It is faced with two problems: the adolescent whose behavior is explained is included in the calculation of the predictor variable (ego-bias problem) and the mutual influence between the adolescent and his classmates is neglected (simultaneity problem). Both problems may lead to inflated effect statistics. The present work introduces potential remedies and illustrates their application at the 
example of adolescent shoplifting activity. Both instrumental-variables regression and non-recursive structural equation models reveal significant effects of classmates' delinquency rate on young people's shoplifting involvement.

Keywords: Delinquent peers, instrumental-variables analysis, shoplifting

\section{Problemstellung}

Peer-Effekten kommt in der kriminologischen Forschung eine große Bedeutung zu. Zahlreichen Untersuchungen zufolge erhöht die Anzahl der Kontakte zu delinquenzaffinen Gleichaltrigen die Wahrscheinlichkeit eigener strafbarer Handlungen (Gerstner \& Oberwittler 2015; Hirtenlehner \& Bacher 2017; Hoeben, Meldrum, Walker \& Young 2016; Matsueda \& Anderson 1998; Pratt et al. 2010; Svensson \& Oberwittler 2010; Warr 2002). Zweifellos repräsentiert Peerdelinquenz eines der robustesten Korrelate selbstberichteter Kriminalität.

Die Schätzung von Peer-Effekten setzt eine Messung der Peerdelinquenz voraus. Zwei grundlegende Ansätze der Erfassung delinquenter Aktivitäten im Freundeskreis lassen sich unterscheiden (Gerstner \& Oberwittler 2015): indirekte Messungen, bei denen die Befragten Auskunft über die (wahrgenommene) Kriminalität ihrer Peers geben, und direkte Messungen, bei denen die Kriminalitätsbeteiligung der Peers bei den Freunden selbst erhoben wird. Indirekte Messungen haben den Nachteil, dass sie den Peer-Effekt systematisch überschätzen (Haynie \& Osgood 2005; Hoeben et al. 2016; Rebellon \& Modecki 2014), da das eigene Verhalten auf die Freunde projiziert und/ oder irrtümlicherweise eine Ähnlichkeit mit dem Verhalten der Freunde angenommen wird. Mit der Überbewertung des Peer-Effektes ist häufig auch eine Unterschätzung der Erklärungskraft anderer Prädiktoren des Legalverhaltens verbunden.

Aus den genannten Gründen wird oft ein direkter Weg der Erfassung umgebender Peerdelinquenz bevorzugt, in dem z. B. die "peer-berichtete« Kriminalitätsbelastung der Freunde als unabhängige Variable in statistische Zusammenhangs- und Kausalanalysen einbezogen wird. Aufgrund von Restriktionen in der Datenerhebung wird in solchen Fällen gerne die aus den Selbstangaben der Klassenkameraden errechnete Kriminalitätsprävalenzrate der Schulklasse, die der Jugendliche besucht, als Indikator für das Niveau der Peerdelinquenz verwendet. Manchmal werden auch die individuellen Kriminalitätsinzidenzen der Schüler auf Klassenebene gemittelt. Die Ergebnisse dieser Studien zeigen durchwegs, dass die Kriminalitätswahrscheinlichkeit eines Schülers steigt, wenn die Delinquenzrate in seiner Klasse wächst (Hirtenlehner \& Bacher 2017; Kim \& Fletcher 2018; Weiss 2019; Windzio 2013).

Das Ablesen des Umfangs delinquenter Peerexposition aus der einschlägigen Täterrate in der eigenen Schulklasse geht von den Annahmen aus, dass sich (1.) die Freunde eines Jugendlichen aus dem Pool der Klassenkameraden rekrutieren und dass sich (2.) die Kriminalitätsrate einer Schulklasse als Messgröße für eine unverzerrte Schätzung des Peer-Effektes eignet. Die erste Annahme soll hier nicht problematisiert werden und vereinfachend daran festgehalten werden, dass die Freunde auch Klassenkameraden sind. Inhaltlich unbestritten bleibt, dass eine gemeinschaftliche Verstrickung in Kriminalität auch Gleichaltrige außerhalb des Klassenverbandes involvieren kann und nicht mit allen Freunden die Schulklasse geteilt wird (Friemel \& Knecht 2009; Gerstner \& Oberwittler 2015; Oberwittler 2004). Im Fokus des vorliegenden Beitrages steht hingegen die zweite Annahme, nämlich dass die aus den Selbstauskünften der einzelnen Schüler hochaggregierte Klassenkriminalitätsrate eine brauchbare Maßzahl für die Bestimmung des Einflusses der Kontakte zu delinquenten Peers auf das eigene Legalverhalten darstellt. Wie unschwer zu erkennen ist, haften der aus den Angaben der Schüler berechneten Delinquenzrate der Schulklasse zwei grundlegende methodische Probleme an. Wir wollen eines als Ego-Bias- und das andere als Reziprozitäts- oder Simultanitätsproblem bezeichnen ${ }^{1}$. Beide ziehen im Falle einer Nicht-Berücksichtigung bei der Datenanalyse das Risiko einer erheblichen Überschätzung delinquenter PeerEffekte nach sich. Während aber Zweiterem auch durch ein Panel-Design begegnet werden könnte, würde eine mehrmalige Befragung desselben Personenkreises aufgrund der dokumentierten Stabilität delinquenten Handelns (frühere Kriminalität ist der beste Prädiktor späterer Kriminalität) nur wenig an der Virulenz des Ersteren än-

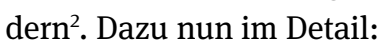

(1.) In die Schätzung der Delinquenzrate einer Schulklasse fließt die Delinquenz des Jugendlichen, dessen kriminelles Handeln als Zielvariable Gegenstand der Untersuchung ist, mit ein. Damit ist eine grundlegende An-

1 Synonym wird manchmal auch vom Bidirektionalitäts- oder Endogenitätsproblem gesprochen.

2 Beide Probleme stellen sich auch bei der Bestimmung des Einflusses der aus Selbstauskünften der Befragten hochaggregierten Kriminalitätsrate im Wohnviertel auf das persönliche Legalverhalten - bei der Analyse von Quartierseffekten freilich etwas entschärft durch eine gesteigerte Größe der Kontexteinheiten. 
nahme jeglicher Kausalforschung verletzt, nämlich dass Ursache und Wirkung getrennt gemessen werden sollen: Das untersuchte (delinquente) Verhalten eines Jugendlichen ist sowohl abhängige Variable (Wirkung) als auch unabhängige Variable (Ursache), da es in die Berechnung der Kriminalitätsrate einer Schulklasse originär eingeht. Wir wollen dieses Problem als Ego-Bias-Problem bezeichnen und nachfolgend die sich daraus ergebenden fatalen Konsequenzen für die Schätzung des Peer-Effektes aufzeigen, um dann praktikable Modellierungsalternativen vorstellen $\mathrm{zu}$ können. Die präferierte Lösung des Ego-Bias-Problems impliziert, dass für die Schätzung des Peer-Effektes nicht die einfach auf Klassenebene hochaggregierte Prävalenzrate (oder die gemittelte Kriminalitätsinzidenz) verwendet wird, sondern der um den Jugendlichen, dessen kriminelles Handeln analysiert wird, bereinigte Täteranteil (oder Inzidenzmittelwert). In die Berechnung der Expositionsvariablen sollten nur die Klassenkameraden (alteri) einbezogen werden. Daraus resultieren allerdings unterschiedliche Werte der Expositionsvariablen für die Schüler einer Klasse, was deren Verwendung als Kontextmerkmal in einer statistischen Mehrebenenanalyse (Snijders \& Bosker 2004) unmöglich macht.

(2.) Aber auch nach der Bereinigung um den Ego-Bias verbleibt noch ein zweites Problem, das darin besteht, dass der Jugendliche, dessen strafbares Verhalten als abhängige Variable untersucht wird, selbst einen Einfluss auf das delinquente Handeln seiner Schulkameraden haben kann. Nicht nur die Mitschüler lenken den Befragten, dieser übt auch seinerseits eine gestaltende Kraft auf das Legalverhalten der Klassenkameraden aus. Es liegt somit eine reziproke bzw. bidirektionale Wirkungsdynamik vor. Die sich daraus ergebenden Schwierigkeiten für die Bestimmung des kriminogenen Peer-Effektes sollen im Weiteren als Reziprozitäts- bzw. Simultanitätsproblem bezeichnet werden. Die Tatsache, dass auch die ego-korrigierte Delinquenzrate der Schulklasse vom Handeln des Befragten beeinflusst wird, verletzt mit der Exogenitätsbedingung eine zentrale Annahme aller Regressionsverfahren, nämlich dass der Fehlerterm der abhängigen Variablen nicht mit den Werten der unabhängigen Variablen korreliert (Bushway \& Apel 2010).

Der vorliegende Beitrag greift die angesprochenen Problematiken auf, um ihre Konsequenzen zu illustrieren und praktikable Lösungsangebote zu unterbreiten. Nachfolgend sollen zunächst in Abschnitt 2 die Folgen der logischen Abhängigkeit der aggregierten Klassenkriminalitätsrate vom Legalverhalten des betrachteten Schülers anhand eines einfachen Rechenbeispiels mit fiktiven Daten verdeutlicht werden. Anschließend stellt Abschnitt 3 Lö- sungsansätze für die skizzierten Problemfelder in ihren methodologischen Grundlagen dar. Besondere Beachtung finden dabei die Korrektur der Expositionsvariablen um die Kriminalitätsbeteiligung des einzelnen Schülers und die statistische Modellierung des Peer-Effektes mittels Instrumentalvariablenregressionsanalysen und nicht-rekursiven Strukturgleichungsmodellen. Die vorgestellten Abhilfestrategien werden dann in Abschnitt 4 exemplarisch implementiert und evaluiert. Das Anwendungsbeispiel befasst sich mit der Bedeutung der Ladendiebstahlsdelinquenz der Klassenkameraden für die eigene Einlassung auf die Entwendung von Waren aus Verkaufsräumen. Eine Zusammenfassung der wesentlichen Erkenntnisse (Abschnitt 5) schließt den Beitrag ab.

\section{Ein einfaches Beispiel zur Illustration der Ego-Bias-Problematik}

Um ein erstes Verständnis der aus dem Ego-Bias-Problem resultierenden Verzerrungen zu gewinnen, wird ein einfaches lineares Wahrscheinlichkeitsmodell für die Prävalenz delinquenten Verhaltens untersucht. Die Zielvariable differenziert also nur zwischen dem Vorkommen oder Nicht-Vorkommen von Kriminalität. Das Modell nimmt an, dass die Wahrscheinlichkeit für das Begehen einer delinquenten Handlung nur von einem dichotomen Risikofaktor $x$ mit den Werten »1« für »vorhanden« und »0« für »nicht vorhanden « abhängt. Fehlt der Risikofaktor, ist die Tatbegehungswahrscheinlichkeit $b_{0}$, ist er existent, besitzt die Tatbegehungswahrscheinlichkeit den Wert $b_{0}+b_{1}$. Das entsprechende Modell lautet:

$$
E\left(y_{i k}\right)=E\left(P\left(Y_{i k}=1\right)\right)=b_{0}+b_{1} \cdot x_{i k},
$$

wobei $x_{i k}$ der Wert des Risikofaktors des Schülers $i$ in der Klasse $k$ ist. Ein Kontexteffekt (z. B. ein Einfluss des Legalverhaltens der Klassenkameraden) liegt nicht vor. Die Wahrscheinlichkeit einer delinquenten Handlung hängt ausschließlich vom individuellen Risikofaktor $x$ (und eventuell weiteren nicht erfassten individuellen Merkmalen) ab.

Wir wollen nun annehmen, dass jede Klasse nur aus zwei Schülern besteht, von denen ein Schüler den Risikofaktor aufweist und der andere nicht. Insgesamt liegen Informationen für zehn Klassen vor. Tabelle 1 stellt die Datenkonstellation dar, wobei für die Regressionsparameter Werte von $b_{0}=0,1$ und $b_{1}=0,5$ festgelegt wurden. 
Tabelle 1: Einfaches Datenbeispiel zur Verdeutlichung der Ego-Bias-Problematik

\begin{tabular}{|c|c|c|c|c|c|}
\hline Kla & Schüler & $\begin{array}{l}\text { Risiko- } \\
\text { faktor x }\end{array}$ & $\begin{array}{l}\text { Erwartungswert } \\
\text { des delinquenten } \\
\text { Verhaltens y }\end{array}$ & $\begin{array}{l}\text { Beobachteter } \\
\text { Wert von y }\end{array}$ & $\begin{array}{l}\text { Täterrate } \\
\text { in der } \\
\text { Klasse }\end{array}$ \\
\hline 1 & 1 & 1 & 0,6 & 1 & 1,0 \\
\hline 1 & 2 & 0 & 0,1 & 1 & 1,0 \\
\hline 2 & 3 & 1 & 0,6 & 1 & 0,5 \\
\hline 2 & 4 & 0 & 0,1 & 0 & 0,5 \\
\hline 3 & 5 & 1 & 0,6 & 1 & 0,5 \\
\hline 3 & 6 & 0 & 0,1 & 0 & 0,5 \\
\hline 4 & 7 & 1 & 0,6 & 1 & 0,5 \\
\hline 4 & 8 & 0 & 0,1 & 0 & 0,5 \\
\hline 5 & 9 & 1 & 0,6 & 1 & 0,5 \\
\hline 5 & 10 & 0 & 0,1 & 0 & 0,5 \\
\hline 6 & 11 & 1 & 0,6 & 1 & 0,5 \\
\hline 6 & 12 & 0 & 0,1 & 0 & 0,5 \\
\hline 7 & 13 & 1 & 0,6 & 0 & 0,0 \\
\hline 7 & 14 & 0 & 0,1 & 0 & 0,0 \\
\hline 8 & 15 & 1 & 0,6 & 0 & 0,0 \\
\hline 8 & 16 & 0 & 0,1 & 0 & 0,0 \\
\hline 9 & 17 & 1 & 0,6 & 0 & 0,0 \\
\hline 9 & 18 & 0 & 0,1 & 0 & 0,0 \\
\hline 10 & 19 & 1 & 0,6 & 0 & 0,0 \\
\hline 10 & 20 & 0 & 0,1 & 0 & 0,0 \\
\hline
\end{tabular}

Die Daten wurden so konstruiert, dass die beobachteten Durchschnittswerte erwartungstreue Schätzungen darstellen. Es gilt also:

$$
\begin{gathered}
\bar{y}_{x=0}=E(y / x=0)=b_{0}=0,1 \\
\bar{y}_{x=1}=E(y / x=1)=b_{0}+b_{1}=0,6 \\
b_{0}=0,1 \\
b_{1}=0,5
\end{gathered}
$$

Die Durchführung einer linearen Regression erbringt die erwarteten Ergebnisse (siehe »Modell ohne Peereffekt« in Tabelle 2). Die Konstante und der Steigungskoeffizient zeigen die vorab festgelegten Werte.
Tabelle 2: Ergebnisse einer linearen Regression für die Daten der Tabelle 1

\begin{tabular}{lllll}
\hline & \multicolumn{2}{l}{ Modell ohne Peereffekt } & \multicolumn{2}{l}{$\begin{array}{l}\text { Modell mit Klassen- } \\
\text { delinquenzrate }\end{array}$} \\
\hline $\begin{array}{l}\text { Unabhängige } \\
\text { Variable }\end{array}$ & $\mathrm{b}$ & $\mathrm{p}$ & $\mathrm{b}$ & $\mathrm{p}$ \\
\hline Konstante & $+0,100$ & 0,470 & $-0,250$ & 0,034 \\
\hline Risikofaktor $\mathrm{x}$ & $+0,500$ & 0,018 & $+0,500$ & 0,001 \\
\hline $\begin{array}{l}\text { Delinquenzrate } \\
\text { in der Klasse }\end{array}$ & - & - & $+1,000$ & 0,005 \\
\hline
\end{tabular}

$\mathrm{b}=$ Regressionskoeffizient, $\mathrm{p}=\alpha$-Fehlerniveau

Ergebnisse von OLS Regressionen. Auf eine Mehrebenenanalyse wurde verzichtet, da eine Kontexteinheit nur von 2 Personen gebildet wird. Die Signifikanz der Delinquenzrate der Klasse wurde korrigiert, indem als Fallzahl die Zahl der Klassen und nicht die Zahl der Personen verwendet wurde. Der signifikante Effekt der Delinquenzrate ist auch nach dieser Korrektur vorhanden.

Wird nun das Modell um einen Peer-Effekt erweitert und dieser mittels der aggregierten Delinquenzrate in der Klasse (siehe Spalte »Täterrate« in Tabelle 1) geschätzt, wird ein signifikanter Peer-Effekt von $b_{2}=1,00$ berechnet (siehe »Modell mit Klassendelinquenzrate« in Tabelle 2). Diesen Effekt würden wir vermutlich inhaltlich als kriminalitätserhöhende Wirkung delinquenter Peerexposition interpretieren, obwohl er realiter nicht vorhanden ist. Damit macht das Beispiel deutlich, dass der simple Weg, den kriminogenen Peer-Effekt aus der Kriminalitätsprävalenzrate einer Schulklasse (oder der mittleren Kriminalitätsinzidenz) abzuleiten, in die Irre führen kann. Dabei kann ein signifikanter delinquenter Peer-Effekt ermittelt werden, der de facto nicht besteht. Kurzgefasst: Ein einfaches Hochaggregieren individueller Kriminalitätsdaten auf Schulklassenebene mag artifizielle Peereinflüsse hervorbringen.

\section{Probate Analysestrategien}

In dem oben dargestellten Beispiel wurden die Konsequenzen des Ego-Bias-Problems verdeutlicht. Dieses erwächst daraus, dass der Wert des Schülers in der abhängigen Variablen auch in die Berechnung der unabhängigen Variablen (der Delinquenzrate oder der mittleren Kriminalitätsinzidenz einer Schulklasse) miteinfließt. Eine intuitive Lösung der Problematik besteht darin, den Befragten aus der Bildung der Expositionsvariablen auszuschließen, indem nicht der einfach hochaggregierte Klassendurchschnitt 


$$
\bar{y}_{k}=\frac{1}{n_{k}} \sum_{i=1}^{n_{k}} y_{i k} \quad \text { mit }
$$

$n_{k}=$ Größe der Klasse $k$

$y_{i k}=$ Wert des Schülers $i$ der Klasse $k$ in der abhängigen Variablen $y$

als Prädiktor zur Schätzung des Peer-Effekts verwendet wird, sondern der um Ego, den Schüler $i$, korrigierte Durchschnittswert (ego-korrigierte Klassenkriminalität). Die ego-korrigierte Delinquenzbelastung einer Schulklasse errechnet sich für jeden Schüler aus den Selbstauskünften allein der Klassenkameraden

$$
\bar{y}_{k-i}=\frac{1}{n_{k}-1} \sum_{\substack{j=1 \\ j \neq 1}}^{n_{k}} y_{j k}
$$

und fällt somit für jeden Schüler unterschiedlich aus. Die Bereinigung um den Ego-Bias impliziert, dass verschiedene Schüler ein und derselben Klasse ungleiche Messwerte der delinquenten Peerexposition im Klassenverband aufweisen. Damit liegt allerdings keine für eine statistische Mehrebenenanalyse taugliche Kontextvariable mehr vor ${ }^{3}$. Ego-korrigierte Klassenkriminalität muss als Individualvariable behandelt werden.

Auch nach Beseitigung des Ego-Bias verbleibt indes das Reziprozitäts- oder Simultanitätsproblem. Nicht nur wird Ego von seinen Klassenkameraden beeinflusst, sondern er beeinflusst mit seinem Handeln auch ebendiese.

Das Simultanitätsproblem wurde $u$.a. in einem oft zitierten Beispiel aus der Statuserwerbsforschung von Duncan, Haller \& Portes (1968) ausführlich behandelt. Duncan und Kollegen untersuchten die beruflichen Ansprüche eines Jugendlichen in Abhängigkeit von den beruflichen Ansprüchen seines besten Freundes und weiteren Kovariaten. Die Autoren nehmen an, dass sich die beiden Jugendlichen in ihren beruflichen Ansprüchen gegenseitig beeinflussen. Zusätzlich wird von einem Effekt des sozioökonomischen Status der Eltern und der Intelligenz des Jugendlichen ausgegangen, wobei vermutet wird, dass der sozioökonomische Status der Eltern sowohl auf die beruflichen Ansprüche des eigenen Kindes als auch auf jene des Freundes wirkt. Bei der Intelligenz wird von keiner gegenseitigen Beeinflussung ausgegangen: Die Intelligenz eines Jugendlichen formt nur seine eigenen beruflichen Ansprüche und nicht jene des Freundes. Das von

3 Mehrebenenmodelle setzen voraus, dass ein Kontextmerkmal nur zwischen den Kontexteinheiten, aber nicht innerhalb dieser variiert (Snijders \& Bosker 2004). den Autoren untersuchte Pfadmodell ist schematisch in Abbildung 1 angeführt.

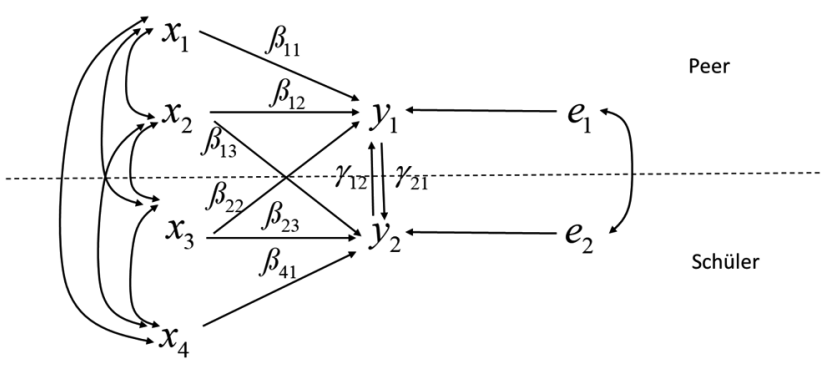

Abbildung 1: Formale Struktur des nicht-rekursiven Pfadmodells von Duncan et al. (1968)

$x_{1}=$ Intelligenz des Freundes,

$x_{2}=$ sozioökonomischer Status der Eltern des Freundes,

$x_{3}=$ sozio-ökonomischer Status der Eltern des Jugendlichen,

$x_{4}=$ Intelligenz des Jugendlichen,

$y_{1}=$ berufliche Ansprüche des Freundes,

$y_{2}=$ berufliche Ansprüche des Jugendlichen,

$e_{1}=$ Fehlerterm für $y_{1}$,

$e_{2}=$ Fehlerterm für $y_{2}$.

In Abbildung 1 bilden die $x$-Variablen die exogenen Variablen, die im Unterschied zu den endogenen $y$-Variablen nicht von anderen Variablen im Modell beeinflusst werden. Die Variable $y_{1}$ entspricht den beruflichen Ansprüchen des Freundes, die Variable $y_{2}$ den beruflichen Ansprüchen des untersuchten Jugendlichen. $x_{1}$ ist die Intelligenz des Freundes, $x_{4}$ jene des untersuchten Jugendlichen. Der sozioökonomische Status der Eltern des Freundes wird durch die Variable $x_{2}$ erfasst, jener der Eltern des untersuchten Jugendlichen durch $x_{3}$. Entsprechend den Modellannahmen gestaltet $x_{1}$ nur $y_{1}$ und $x_{4}$ nur $y_{2}$. Zwischen $y_{1}$ und $y_{2}$ besteht eine gegenseitige Beeinflussung. Die zu schätzenden Modellparameter sind die Effekte $\beta_{i j}$ der exogenen Variablen $x_{1}$ bis $x_{4}$ sowie die reziproken bzw. simultanen Effekte $\gamma_{12}$ und $\gamma_{21}$

Technisch betrachtet stellt im obigen Beispiel die Intelligenz eine Instrumentenvariable dar. Instrumentenvariablen spielen in der ökonomischen Forschung seit langem eine zentrale Rolle und die Instrumentenvariablenschätzung wird in jedem ökonometrischen Lehrbuch (Angrist \& Pischke 2009; Cameron \& Trivedi 2010; Johnston 1984) ausführlich erörtert. Die Idee dazu wurde erstmals von Philipp Wright, einem Ökonomen, in den 1920er Jahren - vermutlich gemeinsam mit seinem Sohn Sewall Wright - formuliert (Stock \& Trebbi 2003). Sewall Wright gilt zugleich als Erfinder der Pfadanalyse, auf der die Ausführungen von Duncan et al. (1968) basieren.

Instrumentenvariablen finden zunehmend auch in der kriminologischen Forschung Beachtung (Angrist 2006; 
Bollen 2012; Bushway \& Apel 2010; Kim \& Fletcher 2018). Sie können in nicht-experimentellen Forschungen zur Behandlung unterschiedlicher Problematiken eingesetzt werden (Bollen 2012; Bushway \& Apel 2010), u. a. zur Lösung des genannten Simultanitätsproblems, also der wechselseitigen Beeinflussung von zwei Variablen, in Abbildung 1 eben $y_{1}$ und $y_{2}$. Mit Bezug auf unsere Ausgangsfragestellung könnte $y_{2}$ die Delinquenz des Schülers $i$ sein und $y_{1}$ die (ego-korrigierte) Delinquenzrate $\bar{y}_{k-i}$ der Klassenkameraden.

Eine Schätzung des Einflusses von $y_{1}$ auf $y_{2}$ ist möglich, wenn für $y_{1}$ eine Instrumentenvariable gefunden wird. Diese muss folgende Bedingungen erfüllen (z.B. Bascle 2008; Bushway \& Apel 2010; Lousdal 2018):

1. Die Instrumentenvariable wirkt direkt auf $y_{1}$ ein, ist also in der Lage, $y_{1}$ zu erklären (Relevanzbedingung). Diese Bedingung kann auch dahingehend abgeschwächt werden, dass die Instrumentenvariable mit der Variablen, deren Prädiktor sie darstellt, stark korreliert.

2. Die Instrumentenvariable von $y_{1}$ wirkt nur indirekt über $y_{1}$ auf $y_{2}$ ein (Exklusionsbedingung), nicht aber direkt. Eine gewisse Korrelation zwischen der Instrumentenvariable und $y_{2}$ ist damit freilich zulässig.

3. Die Instrumentenvariable von $y_{1}$ ist eine »echte« exogene Variable (Exogenitätsbedingung), d. h. sie wird von keinen anderen Variablen des Modells beeinflusst und ist daher mit dem Fehlerterm $e_{2}$ von $y_{2}$ unkorreliert. $^{4}$

Mitunter wird noch als weitere vierte Bedingung die Homogenität der Wirkung der durch die Instrumentenvariable gemessenen Variablen auf die interessierende abhängige Variable angeführt (Lousdal 2018). Damit ist gemeint, dass der Effekt der untersuchten unabhängigen Variablen in unterschiedlichen Teilpopulationen des Samples gleich groß ausfällt. Diese Annahme wird aber allgemein in jeder Regressionsanalyse getroffen und ist somit kein Spezifikum der Verwendung von Instrumentenvariablen.

In Abbildung 1 erfüllt $x_{1}$ diese Bedingung für $y_{1}$. Die Instrumentenvariable wirkt nur auf $y_{1}$ ein. Eine direkte nicht durch $y_{1}$ hergestellte - Wirkung auf $y_{2}$ liegt nicht vor und es besteht auch keine korrelative Beziehung mit $e_{2}$.

Für eine Variable kann auch mehr als eine Instrumentenvariable spezifiziert werden. Das Modell der Abbil-

4 Diese Bedingung wird in der Literatur unterschiedlich bezeichnet. Lousdal (2018) nennt folgende Begriffe: »exchangeability assumption «, »independence assumption «, »ignorable treatment assignment « und »no confounding for the effect of $\mathrm{Z}$ on $\mathrm{Y}$ «. dung 1 könnte also um weitere Instrumentenvariablen $x_{5}$, $x_{6}, \ldots$ erweitert werden, die direkt nur auf $y_{1}$ einwirken.

Ist man nur an der Schätzung des Peer-Effekts (in dem Beispiel die Wirkung von $y_{1}$ auf $y_{2}$ ) interessiert, genügt die Spezifikation von Instrumentenvariablen für $y_{1}$. Wird dagegen die Schätzung beider Effekte (also von $y_{1}$ auf $y_{2}$ und von $y_{2}$ auf $y_{1}$ ) angestrebt, müssen für beide sich wechselseitig beeinflussende Variablen Instrumentenvariablen definiert werden.

Die Bestimmung von Instrumentenvariablen markiert eine inhaltliche Aufgabe und muss theoriegeleitet erfolgen. Formal sollten die ausgewählten Instrumentenvariablen hinsichtlich der Variablen, deren Instrumente sie darstellen, eine hohe Varianzaufklärungskraft bzw. Korrelation aufweisen. ${ }^{5}$ Ist dies der Fall, wird von »starken « Instrumentenvariablen gesprochen, anderenfalls von "schwachen" Instrumentenvariablen.

Zur Prüfung der Relevanzbedingung wird ein F-Test vorgeschlagen (siehe dazu später). Die beiden anderen Bedingungen lassen sich nur bedingt statistisch testen (siehe ebenfalls Abschnitt 4).

Nach der Auswahl von Instrumentenvariablen kann mit der eigentlichen Schätzung des Peer-Effekts begonnen werden. ${ }^{6}$ Dafür bestehen zwei Möglichkeiten:

1. Schrittweise Schätzung im Rahmen der traditionellen Instrumentalvariablenanalyse (als Abfolge zweier aufeinander aufbauender Regressionsanalysen) oder

2. Simultanschätzung im Rahmen eines non-rekursiven Strukturgleichungsmodells (der Einschluss von Instrumentenvariablen wird hier für die Herstellung von Identifizierbarkeit benötigt).

5 Bollen (2012) unterscheidet drei Gruppen von Instrumentenvariablen. Die von ihm als »auxiliary instrumental variables« bezeichnete Gruppe entspricht dem hier gewählten Vorgehen. Es werden aus den Daten zusätzliche Variablen als Instrumentenvariablen ausgewählt. Daneben ist es nach ihm noch möglich, modellbasierte Instrumentenvariablen zu spezifizieren oder diese aufgrund eines (Quasi-)Experiments zu selektieren. Ein Beispiel für letztere Methode ist der Einsatz von Zwillingsdaten.

6 Vor der Schätzung der gesuchten Modellparameter ist allgemein die Identifikation des spezifizierten Modells zu beleuchten (Bushway \& Apel 2010). Mit Identifikation ist gemeint, dass die gesuchten unbekannten Modellparameter als Funktion der empirisch bekannten Modellparameter ausgedrückt werden können. Zum Nachweis der Identifikation wurden unterschiedliche Regeln entwickelt. Für das hier behandelte Problem der Schätzung eines Peer-Effekts ist die Identifikation dann gegeben, wenn für die ego-bereinigte Delinquenzrate der Klassenkameraden mindestens eine Instrumentenvariable spezifiziert werden kann. Soll zusätzlich der Einfluss des kriminellen Handelns des betrachteten Jugendlichen auf die Delinquenzrate seiner Schulkameraden geschätzt werden, muss auch für die Kriminalität des Jugendlichen eine Instrumentenvariable definiert werden. 
Beide Varianten werden im Anwendungsbeispiel implementiert und sollen daher nachfolgend kurz beschrieben werden.

Bei der schrittweisen Schätzung, die ab nun als Instrumentalvariablenanalyse oder Instrumentalvariablenregression bezeichnet werden soll, wird zunächst in einem ersten Schritt eine Regressionsanalyse für $y_{1}$ durchgeführt, in welche als unabhängige Variablen die für $y_{1}$ definierten Instrumentenvariablen und weitere Kovariaten einbezogen werden. In der Literatur wird diesbezüglich empfohlen, alle Kovariaten, die auf $y_{2}$ direkt einwirken, auch in der »first-stage equation « als Prädiktoren zu verwenden (Bascle 2008) ${ }^{7}$. Auf der Grundlage dieser ersten Regression werden für $y_{1}$ erwartete Variablenwerte (Prognosewerte) berechnet. Wenn wir aus Gründen der Vereinfachung das Subskript $i$ weglassen, lautet die Gleichung zur Berechnung der Erwartungswerte mit Blick auf Abbildung 1 wie folgt:

$$
\hat{y}_{1}=\hat{\delta}_{10}+\hat{\delta}_{11} \cdot x_{1}+\hat{\delta}_{12} \cdot x_{2}+\hat{\delta}_{13} \cdot x_{3}+\hat{\delta}_{14} \cdot x_{4}
$$

Entsprechend der Empfehlung in der Literatur werden neben der Instrumentenvariable $x_{1}$ von $y_{1}$ die Kovariaten $x_{2}$ bis $x_{4}$ in die Schätzung einbezogen.

In einem zweiten Schritt wird eine Regressionsanalyse für $y_{2}$ durchgeführt, wobei als unabhängige Variablen die prognostizierten Werte $\hat{y}_{1}$ von $y_{1}$ sowie die anderen vermuteten Kovariaten einbezogen werden. In Bezug auf Abbildung 1 lautet die Schätzgleichung:

$$
y_{2}=\beta_{20}+\beta_{22} \cdot x_{2}+\beta_{23} \cdot x_{3}+\beta_{24} \cdot x_{4}+\gamma_{21} \cdot \hat{y}_{1}+e_{2}
$$

Die Instrumentenvariable $x_{1}$ von $y_{1}$ kommt hier nicht vor, da für sie keine direkte Wirkung auf $y_{2}$ angenommen wird. Als Regressor wird stattdessen $y_{1}$ verwendet, der aus der Instrumentenvariable prognostizierte (exogene) Teil der Streuung von $\hat{y}_{1}$.

Das dargestellte schrittweise Vorgehen führt zu verzerrten, aber konsistenten Schätzwerten des Peer-Effekts, wenn die oben genannten drei Bedingungen erfüllt sind (Bascle 2008; Baum, Schaffer \& Stillman 2003; 2007; Johnston 1984). Konsistent bedeutet, dass der geschätzte Effekt sich mit größer werdender Stichprobe dem wahren Wert annähert. Das Ausmaß der Verzerrung hängt u.a.

7 Diese Forderung wird oft nicht näher begründet. Manchmal wird sie auch nicht als mögliche Option dargestellt, sondern als unbedingt zu wahrende Vorgehensweise präsentiert. von der Erklärungskraft der Instrumentenvariable(n) und der Stichprobengröße ab (Bascle 2008). Für valide inferenzstatistische Schlussfolgerungen ist wie bei der gewöhnlichen Regression Homoskedastizität, Unkorreliertheit und Normalverteilung der Fehlerterme erforderlich (Bascle 2008; Baum et al. 2007). Allerdings stehen robuste Berechnungsverfahren zur Verfügung (siehe dazu später), die Verletzungen dieser Annahmen zulassen.

Zur Durchführung der schrittweisen Schätzung wurden unterschiedliche Verfahren entwickelt. Am populärsten ist die sogenannte Two-Stage-Least-Squares-Technik (TSLS, 2SLS), bei der auf die Kleinste-Quadrate-Methode zurückgegriffen wird. Daneben sind noch weitere Kleinste-Quadrate-Methoden und Maximum-Likelihood-Verfahren verfügbar (Staiger \& Stock 1997). Die sogenannte Limited-Maximum-Likelihood-Methode resultiert in geringeren Verzerrungen und schneidet bei schwachen Instrumentenvariablen besser ab als die 2SLS-Technik. Bei Verletzung der Homoskedastizitätsannahme und/oder bei korrelierten Residuen wird die Generalized Method of Moments (GMM) empfohlen (Baum et al. 2003; 2007).

Bei der Simultanschätzung mittels eines nicht-rekursiven Strukturgleichungsmodells wird das Gesamtmodell in einem Schritt berechnet, wobei i.d. R. Maximum-Likelihood-Verfahren zum Einsatz kommen. Der Begriff »simul$\tan$ « verweist hier auf den Umstand, dass der Peer-Effekt und der Einfluss des eigenen Handelns auf die Alteri zugleich (wechselseitig auspartialisiert) ermittelt werden. Instrumentenvariablen müssen in die Modellgleichungen aufgenommen werden, um trotz reziproker Wirkungspfade eine Identifizierbarkeit des Gleichungssystems zu erlangen. Die simultane Schätzung führt bei richtiger Spezifikation des Modells zu effizienteren Schätzern als die schrittweise Methode, hat aber den Nachteil, dass sich Fehlspezifikationen in anderen Modellteilen auch auf die Schätzung des Peer-Effekts auswirken können (Williams 2015).

Nach erfolgter Berechnung der Modellparameter ist die Güte der Schätzergebnisse zu beurteilen. Abhängig von der Schätzmethode und dem verwendeten Ansatz (schrittweise vs. simultan) stehen unterschiedliche Maßzahlen zur Bewertung der Ergebnisqualität zur Verfügung. Neben den interessierenden Effekten sollte - sofern möglich - geprüft werden, wie gut die gewählten Instrumentenvariablen die an sie gestellten Bedingungen erfüllen.

Zusammenfassend wird für die Schätzung »direkter « Peer-Effekte bei querschnittlichen Klassenzimmerbefragungen somit folgende Vorgangsweise empfohlen:

1. Verwendung von ego-korrigierten Klassenkriminalitätsmaßen als Indikator für kriminogene Peereinflüsse an Stelle von einfach auf Klassenebene hochaggregierten Prävalenzraten und Durchschnittswerten. 
2. Schätzung des Peer-Effektes mittels schrittweiser Instrumentalvariablenanalysen (wofür gängige Statistikprogramme integrierte Module zur Verfügung stellen - z.B. IVREGRESS oder IVREG2 in STATA) oder simultan im Rahmen eines nicht-rekursiven Strukturgleichungsmodells.

\section{Ein Anwendungsbeispiel aus dem Bereich der Ladendiebstahls- delinquenz junger Menschen}

\subsection{Datenbasis und methodisches Vorgehen}

Die im vorausgehenden Abschnitt entwickelten Modellierungsstrategien sollen nachfolgend auf die von Hirtenlehner \& Bacher (2017) zur Prüfung der Interaktion von Peerdelinquenz und Sanktionsrisikowahrnehmung genutzten Daten einer Dunkelfeldbefragung zur Ladendiebstahlsdelinquenz junger Menschen angewandt und anschließend evaluiert werden. ${ }^{8}$ Ebendort findet sich eine ausführliche Beschreibung des Stichprobendesigns und der verwendeten Operationalisierungen. Hier soll nur kurz festgehalten werden, dass rund 3.000 ober- und niederösterreichische Schüler der Jahrgangsstufen 7 und 8 computergestützt im Klassenverband befragt wurden. Das Sample rekrutiert sich aus insgesamt 184 Schulklassen aus 92 zufällig ausgewählten Schulen. An jeder Schule wurde zufallsbasiert eine 7. und eine 8. Klasse selektiert, in denen dann eine Vollerhebung durchgeführt wurde.

Als abhängige Variable wird die 1-Jahres-Prävalenz des Ladendiebstahls $(1=$ in den letzten zwölf Monaten mindestens eine Ladendiebstahlshandlung verübt, $0=$ in den letzten zwölf Monaten keinen Ladendiebstahl begangen) untersucht, als unabhängige Variablen werden die in Tabelle 3 aufgelisteten Schüler- und Kontextmerkmale eingeführt.

8 Der dort berichtete Interaktionseffekt, wonach Abschreckungswirkungen perzipierter Entdeckungsrisiken sich vorwiegend dann einstellen, wenn man viele delinquente Klassenkameraden hat, zeigt sich auch in einer auf ego-korrigierten Klassenkriminalitätswerten basierenden zweistufigen Instrumentalvariablenregression.
Tabelle 3: Regressoren in den untersuchten Lösungsvarianten

\begin{tabular}{|c|c|c|}
\hline & $\begin{array}{l}\text { Variante 1: } \\
\text { Schrittweise } \\
\text { schätzung } \\
\text { mittels IV }\end{array}$ & $\begin{array}{l}\text { Variante 2: } \\
\text { Simultane } \\
\text { Schätzung } \\
\text { mittels SEM }\end{array}$ \\
\hline \multicolumn{3}{|l|}{ Schülermerkmale i (Ego) } \\
\hline Niedrige Selbstkontrolle $\left(x_{1}\right)$ & ja & ja, IV a) \\
\hline $\begin{array}{l}\text { Niedrige Sanktionsrisikowahrnehmung } \\
\left(x_{2}\right)\end{array}$ & ja & ja, IV a) \\
\hline Niedrige Moralität $\left(x_{3}\right)$ & ja & ja, IV a) \\
\hline Geschlecht $\left(x_{4}\right)(1=$ Junge, $0=$ Mädchen $)$ & ja & ja, IV a) \\
\hline \multicolumn{3}{|l|}{$\begin{array}{l}\text { ego-korrigierte Klassenmerkmale } \\
\text { (Durchschnittswerte allein der } \\
\text { Klassenkameraden) }\end{array}$} \\
\hline Ladendiebstahlsbelastung $\left(y_{1}=\bar{y}_{k-i}\right)$ & ja & ja \\
\hline Niedrige Selbstkontrolle $\left(\bar{x}_{1}=\bar{x}_{1 k-i}\right)$ & ja, nur IV ${ }^{b)}$ & - \\
\hline $\begin{array}{l}\text { Niedrige Sanktionsrisikowahrnehmung } \\
\left(\bar{x}_{2}=\bar{x}_{2 k-i}\right)\end{array}$ & $\mathrm{ja}$, nur IV $^{\mathrm{b})}$ & - \\
\hline Niedrige Moralität $\left(\bar{x}_{3}=\bar{x}_{3 k-i}\right)$ & ja, nur IV ${ }^{\text {b) }}$ & - \\
\hline Anteil Jungen $\left(\bar{x}_{4}=\bar{x}_{4 k i-i}\right)$ & ja, nur IV ${ }^{\text {b) }}$ & - \\
\hline \multicolumn{3}{|l|}{$\begin{array}{l}\text { Weitere Kontextmerkmale auf } \\
\text { Klassenebene }\end{array}$} \\
\hline $\begin{array}{l}\text { Schulform }\left(z_{1}\right)(1=\text { Gymnasium, } \\
\left.0=\text { Sonstige }{ }^{c)}\right)\end{array}$ & ja & ja \\
\hline $\begin{array}{l}\text { Schulstufe }\left(z_{2}\right)(1=8 . \text { Schulstufe, } \\
0=7 . \text { Schulstufe })\end{array}$ & ja & ja \\
\hline
\end{tabular}

a) Instrumentenvariable (IV) für die Delinquenz des Schülers Ego bei der Schätzung mittels Strukturgleichungsmodell

b) Instrumentenvariable (IV) für die Delinquenz der Klassenkameraden

c) Hauptschule bzw. Neue Mittelschule

Bei der Auswahl der unabhängigen Variablen wurde angenommen, dass entsprechend Hirtenlehner \& Bacher (2017) die Delinquenz eines Schülers abgesehen von der Kriminalitätsbelastung der Freunde von der Moralität, der Selbstkontrolle, der Sanktionsrisikowahrnehmung und dem Geschlecht des Individuums abhängt. Da dieses Erklärungsmodell für alle Schüler gilt, also für den untersuchten Schüler Ego und dessen Klassenkameraden, werden auf der Individualebene und der Klassenebene dieselben Einflussfaktoren vermutet. Als Kontextmerkmale auf Klassenebene, die sowohl die Delinquenzrate der Schulkameraden (= ego-korrigierte Klassenkriminalität) als auch das Legalverhalten des betrachteten Schülers beeinflussen, werden die Schulform und die Schulstufe spezifiziert. Durch diese beiden Variablen sollen Selektionseffekte (die nicht-zufällige Verteilung junger Menschen auf die verschiedenen Schultypen) und weitere Sozialisationseffekte 
erfasst werden. ${ }^{9}$ Die Daten wurden mit IBM-SPSS Version 24 aufbereitet. ${ }^{10}$ Fehlende Werte in den unabhängigen Variablen wurden mittels der EM-Methode (Reisinger, Svecnik \& Schwetz 2012) geschätzt. ${ }^{11}$ In der Zielvariablen liegen keine Missing Values vor. Die Syntax zur Berechnung der ego-korrigierten Klassenmerkmale ist im Anhang dokumentiert.

Entsprechend den erörterten Analysestrategien soll zunächst eine schrittweise Schätzung des Peereinflusses mittels Instrumentalvariablenanalyse (Variante 1) vorgenommen werden, daran anschließend dann eine simultane Schätzung der reziproken Wirkungsdynamik mittels Strukturgleichungsmodellierung (Variante 2). Für die Berechnung der Modellparameter werden in beiden Varianten lineare Wahrscheinlichkeitsmodelle eingesetzt. Wir haben uns für einen linearen Modellierungsansatz entschieden, weil die daraus gewonnenen Regressionsgewichte am einfachsten $\mathrm{zu}$ interpretieren sind und methodologische Arbeiten darauf hinweisen, dass logistische Analysen zu identen Befunden führen (Hellevik 2009). Da bezüglich der Prädiktoren gerichtete Hypothesen vorliegen, wird für alle Signifikanztestungen ein einseitiges Fehlerniveau verwendet.

Bei der Auswertung zu berücksichtigen ist jedenfalls, dass a) aufgrund des Stichprobenplans hierarchisch organisierte Daten mit einer Clusterstruktur vorliegen und b) aufgrund der dichotomen Natur der Zielvariablen die Annahme der Homoskedastizität der Fehlerterme automatisch verletzt ist. Aus den genannten Gründen sind spezielle Schätzverfahren zu wählen (siehe unten).

Um das Ausmaß der Verzerrung aufzuzeigen, das aus der Vernachlässigung des Ego-Bias- und des Simultanitätsproblems resultiert, wurde als Ausgangsbasis mit Mplus Version 8.2. (Muthén \& Muthén 2017) ${ }^{12}$ eine Mehrebenenanalyse gerechnet, in welcher entsprechend der bisherigen Praxis die hochaggregierte Klassenkriminalitätsrate (der Anteil der delinquenten Schüler in einer Klasse) als Kontextvariable verwendet wurde. Der Rückgriff auf die Mehrebenenanalyse ist dabei der geschachtelten

\footnotetext{
9 Kim \& Fletcher (2018) verwenden zur Berücksichtigung des Selektionseffektes ein »fixed-effects«-Modell, in das für jede Schule eine Dummy-Variable einbezogen wird. Wir berücksichtigen die durch Selektionsprozesse und die gemeinsame Beschulung entstehende Ähnlichkeit der Schüler einer Klasse durch die Verwendung eines Mehrebenenmodells.

$10 \mathrm{https} / / /$ www.ibm.com/de-de/products/spss-statistics

11 Da sich die fehlenden Werte quantitativ in engen Grenzen halten, wurde auf diese einfachere Methode zurückgegriffen und auf multiple Imputationstechniken verzichtet.

12 https://www.statmodel.com/
}

Datenstruktur geschuldet. Um eine ausreichende Fallzahl auf Klassenebene $\mathrm{zu}$ erhalten, wurden nur Klassen mit mehr als 9 Schülern in die Analyse aufgenommen. ${ }^{13}$ Als Schätzverfahren wurde angesichts der Verletzung der Homoskedastizitätsannahme eine Maximum-LikelihoodMethode mit robusten Standardfehlern (MLR) verwendet. Dabei wurde ein signifikanter Peer-Effekt von $\beta=0,86$ ( $p<0,001$ ) ermittelt (siehe Tabelle 5), der besagt, dass sich bei einem Anstieg der Klassenkriminalitätsrate um $10 \%$ die Delinquenzwahrscheinlichkeit des untersuchten Schülers um 8,58\% erhöhen würde. Dies entspräche einem sehr starken Peereinfluss. Eine solche Interpretation ist allerdings wegen der Vernachlässigung des Ego-Bias- und des Reziprozitätsproblems als inkorrekt zu betrachten.

\subsection{Instrumentalvariablenanalyse}

Mplus 8.2. stellt unseres Wissens kein spezielles Modul für die Instrumentalvariablenanalyse zur Verfügung. Diese kann aber leicht realisiert werden, indem bei der Datenaufbereitung mit SPSS die Erwartungswerte für die egokorrigierte Delinquenzrate der Mitschüler berechnet und als Variable an das Programm übergeben werden. Die vorgeschaltete Regression erbringt in IBM-SPSS Version 24 eine erklärte Varianz von $49,4 \%$, wobei die verwendeten Instrumentenvariablen eine Varianzaufklärungsleistung von 17,6\% aufweisen. Der korrespondierende F-Wert von 162,68 überschreitet den in der Literatur genannten Schwellenwert von 10 (z. B. Baum et al. 2007) um ein Vielfaches. Demzufolge liegen also »starke« Instrumentenvariablen vor, wobei allerdings einschränkend anzumerken ist, dass der F-Test für die vorhandene Datenstruktur nur bedingt anwendbar ist (siehe unten). Eine statistisch korrektere Betrachtung legt den Schluss nahe, dass keine schwachen, aber auch keine allzu starken Instrumente gewählt wurden.

Innerhalb der Instrumentalvariablen besitzt die Selbstkontrolle der Klassenkameraden $(\beta=0,22)$ die höchste Determinationskraft, gefolgt von der Moralität und der Sanktionsrisikowahrnehmung der Mitschüler $(\beta=0,20$ und $\beta=0,14)$.

13 Damit verbleiben 2.807 Personen aus 153 Klassen in der Analyse. Auswertungen für alle Klassen ab 5 befragten Schülern liefern inhaltlich identische Ergebnisse. 
Tabelle 4: Ergebnisse der vorgeschalteten Regressionsanalyse zur Berechnung der prognostizierten Werte der ego-bereinigten Klassendelinquenz

\begin{tabular}{|c|c|c|c|c|}
\hline & b & se(b) & B & $\mathbf{P}$ \\
\hline Konstante & $-0,306$ & 0,016 & & 0,000 \\
\hline \multicolumn{5}{|l|}{ Kovariaten: Schülermerkmale } \\
\hline Niedrige Selbstkontrolle & 0,001 & 0,001 & 0,020 & 0,144 \\
\hline Niedrige Sanktionsrisikowahrnehmung & 0,000 & 0,001 & 0,006 & 0,371 \\
\hline Niedrige Moralität & 0,000 & 0,001 & $-0,005$ & 0,402 \\
\hline Geschlecht (1 = Junge, 0 = Mädchen) & 0,000 & 0,002 & $-0,002$ & 0,451 \\
\hline Schulform (1 = Gymnasium, 0 = Sonstige) & $-0,014$ & 0,002 & $-0,111$ & 0,000 \\
\hline Schulstufe $(1=8$. Stufe, $0=7$. Stufe $)$ & 0,010 & 0,002 & 0,079 & 0,000 \\
\hline \multicolumn{5}{|c|}{ Instrumentenvariablen: ego-korrigierte Klassenmerkmale } \\
\hline Niedrige Selbstkontrolle & 0,014 & 0,001 & 0,217 & 0,000 \\
\hline Niedrige Sanktionsrisikowahrnehmung & 0,009 & 0,001 & 0,136 & 0,000 \\
\hline Niedrige Moralität & 0,006 & 0,001 & 0,195 & 0,000 \\
\hline Anteil Jungen & 0,010 & 0,006 & 0,031 & 0,042 \\
\hline $\mathrm{R}^{2}$ Gesamt & 0,494 & & & \\
\hline F-Wert Gesamt & 90,137 & & & 0,000 \\
\hline $\mathrm{R}^{2}$ der Instrumentenvariablen & 0,176 & & & \\
\hline F-Werte für $\mathrm{R}^{2}$ der Instrumentenvariablen & 162,683 & & & 0,000 \\
\hline
\end{tabular}

$\mathrm{b}=$ unstandardisierter Regresssionskoeffizient,

se(b) = Standardfehler von b,

$\beta=$ standardisierter Regressionskoeffizient,

$\mathrm{p}=\alpha$-Fehler (einseitig)

Datenquelle: Ladendiebstahlsstudie (Hirtenlehner \& Bacher 2017), ungewichtete Daten, alle Klassen mit mehr als 9 befragten Schülern, $\mathrm{n}=2.807$

Tabelle 5: Ergebnisse der Schätzung der unterschiedlichen Modellvarianten zur Bestimmung des kriminogenen Peer-Effekts

\begin{tabular}{lrrrrrrrrr}
\hline & $\begin{array}{r}\text { Vernachlässigung des } \\
\text { Ego-Bias- und des } \\
\text { Simultanitätsproblems }\end{array}$ & $\begin{array}{r}\text { Variante 1a: Manuelle Variante 1b: Integrierte } \\
\text { IV-Schätzung (IBM- }\end{array}$ & $\begin{array}{c}\text { Variante 2: Schätzung } \\
\text { SPSS 24 \& Mplus 8.2.) }\end{array}$ & $\begin{array}{r}\text { Modul IVREG2) } \\
\text { IVïtung (Stata14, }\end{array}$ \\
\hline & Parameter & $\mathrm{p}$ & Parameter & $\mathrm{p}$ & Parameter & $\mathrm{p}$ & Parameter & $\mathrm{p}$ \\
\hline Konstante & 0,010 & 0,0185 & 0,032 & 0,0000 & 0,032 & 0,0000 & 0,033 & 0,0000 \\
\hline Niedrige Selbstkontrolle & 0,024 & 0,0000 & 0,026 & 0,0000 & 0,026 & 0,0000 & 0,026 & 0,0000 \\
\hline Niedrige Sanktionsrisikowahrnehmung & 0,013 & 0,0020 & 0,016 & 0,0005 & 0,015 & 0,0005 & 0,016 & 0,0005 \\
\hline Niedrige Moralität & 0,027 & 0,0000 & 0,031 & 0,0000 & 0,031 & 0,0000 & 0,031 & 0,0000 \\
\hline Geschlecht (1 = Junge, 0 Mädchen) & 0,011 & 0,0690 & 0,014 & 0,0435 & 0,013 & 0,0505 & 0,014 & 0,0041 \\
\hline Peer-Effekt & 0,858 & 0,0000 & 0,206 & 0,0725 & 0,191 & 0,0495 & 0,142 & 0,0270 \\
\hline Schulform & $-0,010$ & 0,0010 & $-0,011$ & 0,1165 & $-0,010$ & 0,0740 & $-0,010$ & 0,0900 \\
\hline Schulstufe & $-0,011$ & 0,0005 & 0,005 & 0,2990 & 0,006 & 0,2235 & 0,007 & 0,1670 \\
\hline
\end{tabular}

Datenquelle: Ladendiebstahlsstudie (Hirtenlehner \& Bacher 2017), ungewichtete Daten, alle Klassen mit mehr als 9 befragten Schülern, $\mathrm{n}=2.807, \mathrm{p}=\alpha$-Fehler (einseitig) 
Übersicht 1: Verfügbare Tests in STATA 14

\begin{tabular}{llll}
\hline Test & Fragestellung & Standardvariante $^{\text {a) }}$ & Robuste Variante $^{\text {b) }}$ \\
\hline Fehlende Identifikation & $\begin{array}{l}\mathrm{H}_{0} \text { : Es liegt keine ausreichende Zahl an } \\
\text { Instrumentenvariablen vor. }\end{array}$ & $\begin{array}{l}\text { Anderson canonical } \\
\text { correlation LM Statistic }\end{array}$ & $\begin{array}{l}\text { Kleibergen-Paap rk LM } \\
\text { Statistic }\end{array}$ \\
\hline Fehlende Relevanz & $\mathrm{H}_{0}$ : Es liegen nur schwache Instrumente vor. & $\begin{array}{l}\text { Cragg-Donald Wald F } \\
\text { Statistic }\end{array}$ & $\begin{array}{l}\text { Kleibergen-Paap rk Wald F } \\
\text { Statistic }\end{array}$ \\
\hline Vorhandene Exogenität & $\begin{array}{l}\mathrm{H}_{0} \text { : Fehlerterme der abhängigen Variablen und } \\
\text { die Instrumentenvariable sind unkorreliert, } \\
\text { gegeben es liegt zumindest eine exogene } \\
\text { Instrumentenvariable vor. }\end{array}$ & Sargan Statistic & Hasen J Statistic \\
& & & \\
\hline
\end{tabular}

a) Bei der Standardvariante wird Homoskedastizität, Unkorreliertheit und identische Verteilung der Fehlerterme für beliebige Ausprägungskombinationen der unabhängigen Variablen angenommen (i.i.d. Bedingung = independent identical distrubuted error terms)

b) Bei der robusten Schätzung werden die Annahmen der Standardvariante nicht getroffen.

Nach Einlesen der prognostizierten ego-korrigierten Mitschülerkriminalitätswerte in Mplus kann der Peereinfluss bestimmt werden. Mplus 8.2. berechnet einen Peereffekt von 0,21 mit einer tendenziellen Signifikanz $(p<0,10)$. Im Vergleich zur bisher verwendeten inkorrekten Schätzung reduziert sich der Effekt deutlich von 0,858 auf 0,206 (siehe Tabelle 5).

Die soeben vorgenommene Analyse mit SPSS und Mplus trägt der Clusterstruktur der Daten und der Heteroskedastizität Rechnung. Sie trifft aber die Annahme, dass die Fehlerterme innerhalb eines Clusters, also einer Schulklasse, unkorreliert sind. Diese Annahme ist insofern problematisch, als der untersuchte Jugendliche in die Berechnung der ego-korrigierten Klassenkriminalitätswerte aller seiner Mitschüler eingeht, sodass klassenintern korrelierte Fehlerterme zu vermuten sind. STATA14 bietet mit IVREG2 (Baum et al. 2003; 2007) ein leistungsstarkes Modul für die Instrumentenvariablenregression an. IVREG2 stellt die sogenannte Generalized Method of Moments (GMM) zur Verfügung, die sowohl korrelierte Fehlerterme innerhalb der Cluster als auch Heteroskedastizität zulässt. Zudem stehen umfangreiche Tests für die Prüfung der Modellannahmen zur Verfügung, nämlich ${ }^{14}$ :

- Tests auf fehlende Identifikation (»underidentification tests«). Mit diesen Tests wird geprüft, ob überhaupt Instrumentenvariablen in ausreichender Zahl vorhanden sind. Die $\mathrm{H}_{0}$-Hypothese lautet, dass dies nicht der Fall ist. Erwünscht sind somit signifikante Abweichungen von der $\mathrm{H}_{0}$-Hyopthese.
- Tests auf fehlende Relevanz (»weak identification tests«). Mit diesen Tests wird geprüft, ob schwache oder starke Instrumentenvariablen vorliegen. Die $\mathrm{H}_{0}$ Hypothese lautet, dass nur schwache Instrumentenvariablen vorliegen, und sie sollte daher verworfen werden.

- Tests auf Exogenität (»overidentification tests«). Mit diesen Tests wird untersucht, ob die Fehlerterme unabhängig von den Instrumentenvariablen sind. Anwendbar sind sie nur, wenn mehr als eine Instrumentenvariable verwendet wird. Erwünscht ist in diesem Fall ein insignifikantes Ergebnis, da die $\mathrm{H}_{0}$-Hypothese lautet, dass die Fehlerterme und die Instrumentenvariablen unkorreliert sind. Vorausgesetzt wird, dass mindestens ein exogenes Instrument vorliegt, wobei sich diese Voraussetzung selbst nicht prüfen lässt.

Übersicht 1 fasst die verfügbaren Tests zusammen.

Führt man die schrittweise Instrumentalvariablenanalyse mit IVREG2 durch, wird ein numerisch zwar etwas kleinerer Peer-Effekt von 0,19 ermittelt, welcher jetzt aber statistisch signifikant ausfällt ( $<<0,05$; siehe Tabelle 5). Wenn man der Struktur der gegebenen Daten adäquat Rechnung trägt, kann damit ein systematischer überzufälliger Peereinfluss nachgewiesen werden. Ein Anstieg der Delinquenzrate der Klassenkameraden um $10 \%$ zieht ein um 1,91\% erhöhtes Kriminalitätsrisiko des untersuchten Schülers nach sich.

Tabelle 6 zeigt die Ergebnisse der einschlägigen Modellprüfung.

14 Wir behandeln hier nur die wesentlichen statistischen Tests. Weitere Testverfahren werden in Baum et al. (2007) erörtert. 
Tabelle 6: Ergebnisse der Modellprüfung für die IV-Schätzung mit IVREG2 in Stata 14

\begin{tabular}{|c|c|c|}
\hline Testgröße & Wert & Signifikanz \\
\hline \multicolumn{3}{|l|}{ Test auf fehlende Identifikation } \\
\hline Kleibergen-Paap rk LM Statistic & 32,58 & 0,000 \\
\hline \multicolumn{3}{|c|}{ Test auf Relevanz der Instrumentenvariablen } \\
\hline Cragg-Donald Wald F Statistic & 162,68 & \\
\hline Kleibergen-Paap rk Wald F Statistic & 11,31 & \\
\hline \multicolumn{3}{|c|}{ Kritische Werte nach Stock-Yogo (2005): } \\
\hline $5 \%$ maximal IV relative bias & 16,85 & \\
\hline $10 \%$ maximal IV relative bias & 10,27 & \\
\hline $20 \%$ maximal IV relative bias & 6,71 & \\
\hline $30 \%$ maximal IV relative bias & 5,34 & \\
\hline $10 \%$ maximal IV size & 24,58 & \\
\hline $15 \%$ maximal IV size & 13,96 & \\
\hline $20 \%$ maximal IV size & 10,26 & \\
\hline $25 \%$ maximal IV size & 8,31 & \\
\hline \multicolumn{3}{|l|}{ Test auf Exogenität } \\
\hline Hansen J Statistic & 0,824 & 0,844 \\
\hline
\end{tabular}

Der Test auf fehlende Identifikation (»underidentification test «) erbringt das positive Ergebnis, dass die $\mathrm{H}_{0}$-Hypothese, wonach keine ausreichende Zahl an Instrumentalvariablen vorliegt, verworfen werden kann.

Der F-Test (»Cragg-Donald Wald F Statistic «) $)^{15}$ zur Beurteilung der Relevanz ist in dem Beispiel nur bedingt interpretierbar, da er von unabhängigen und identisch verteilten Fehlertermen ausgeht (siehe Übersicht 1). Wäre diese Annahme erfüllt, wäre der Test signifikant und die $\mathrm{H}_{0}$-Hypothese, dass nur schwache Instrumentenvariablen vorliegen, könnte verworfen werden. Die »KleibergenPaap rk Wald F Statistic « stellt das robuste Pendant zum F-Test dar. Allerdings fehlen für sie allgemein akzeptierte Schwellenwerte (Baum et al. 2007, 490). Empfohlen wird daher (ebenda), die kritischen Werte von Stock \& Yogo (2005) zu verwenden oder auf den Schwellenwert von 10 zurückzugreifen. Mit Blick auf die von Stock \& Yogo (2005) angeführten Schwellen müssten wir von einem maximalen relativen Schätzfehler von ca. $10 \%$ ausgehen. Der Schwellenwert von 10 wird nur knapp überschritten, was indiziert, dass wir keine starken, aber auch keine allzu schwachen Instrumentenvariablen benutzen.

Der Hansen J-Test schließlich prüft die Exogenitätsbedingung. Erwünscht ist hier ein insignifikantes Ergeb-

15 Dieser Wert ist identisch mit dem von SPSS ermittelten F-Wert für die zusätzliche Erklärungskraft der Instrumentenvariablen (siehe Tabelle 4). nis, welches sich auch einstellt. Vorausgesetzt wird freilich, dass mindestens eine der Instrumentenvariablen exogen ist.

Als Zwischenfazit lässt sich somit festhalten, dass ein signifikanter Peer-Effekt in Erscheinung tritt, wenn in der Schätzung die vorhandene Datenstruktur (Heteroskedastizität und Korreliertheit der Fehler innerhalb der Schulklassen) korrekt berücksichtigt wird. Die Wahrscheinlichkeit, dass ein junger Mensch einen Ladendiebstahl begeht, wächst mit der Ladendiebstahlsbelastung seiner Klassenkameraden.

\subsection{Strukturgleichungsmodellierung}

Alternativ lässt sich der Einfluss der Mitschüler auf das eigene Legalverhalten auch im Rahmen einer linearen Strukturgleichungsmodellierung (SEM) bestimmen. Nichtrekursive Kovarianzstrukturanalysen erlauben es, den Effekt delinquenter Klassenkameraden auf das eigene Handeln und die Wirkung des persönlichen Handelns auf die Kriminalität der Mitschüler - also die reziproke Beziehungsdynamik - in einem Rechengang zu schätzen. Die Berechnung der Modellparameter erfolgte hier wiederum im Maximum-Likelihood-Verfahren mit robusten Standardfehlern. Um das Gleichungssystem identifizierbar zu machen, muss jeweils mindestens eine Instrumentenvariable als Prädiktor der ego-bereinigten Klassenkriminalitätsrate und der Ladendiebstahlsprävalenz des individuellen Schülers spezifiziert werden.

Die Simultanschätzung mittels SEM liefert in Mplus 8.2. einen kriminogenen Peer-Effekt in der Höhe von 0,14, der numerisch zwar kleiner als jener der Instrumentalvariablenregression ausfällt, sich aber ebenfalls statistischer Signifikanz erfreuen darf ( $p<.05$; siehe Tabelle 5). Alle Modellprüfgrößen deuten auf eine sehr gute Anpassungsleistung hin (siehe Tabelle 7), sodass hier der in Kapitel 3 genannte Vorteil der simultanen Schätzung, nämlich deren größere Effizienz, tatsächlich zum Tragen kommt.

Tabelle 7: Modellfit, Residuen und Modifikationsindizes des berechneten Strukturgleichungsmodells

\begin{tabular}{lcr}
\hline Prüfgrößen & Schwellen $^{\text {a) }}$ & $\begin{array}{r}\text { Empirischer } \\
\text { Wert }\end{array}$ \\
\hline Modellfit & & \\
\hline $\begin{array}{l}\text { Root-Mean-Square-Error-of- } \\
\text { Approximation (RMSEA) }\end{array}$ & $<0,05$ & 0,000 \\
\hline Comperative-Fit-Index (CFI) & $>0,95(0,97)$ & 1,000 \\
\hline Tucker-Lewis-Index (TLI) & $>0,95(0,97)$ & 1,092 \\
\hline
\end{tabular}


Tabelle 7: (fortgesetzt)

\begin{tabular}{lcr}
\hline Prüfgrößen & Schwellen $^{\text {a) }}$ & $\begin{array}{r}\text { Empirischer } \\
\text { Wert }\end{array}$ \\
\hline $\begin{array}{l}\text { Standardized-Root-Mean-Square- } \\
\text { Residual (SRMR) }\end{array}$ & $<0,05$ & 0,005 \\
\hline Standardisierte Residuen & $>1,96$ & keine \\
\hline Modifikationsindizes & $>3,84$ & keine \\
\hline
\end{tabular}

a) Die angeführten Akzeptanzschwellen (mit Ausnahme des Modifikationsindizes) stammen aus Geiser (2011, 60-62). Für den Modifikationsindex ergibt sich der Schwellenwert aus der Tatsache, dass er eine Chi-Quadrat-Verteilung mit einem Freiheitsgrad besitzt.

Das empirische Modell ist in Abbildung 2 auch graphisch dargestellt. Wie den Ergebnissen entnommen werden kann, wirkt auf Seite der Schulkameraden nur eine Variable signifikant auf die ego-bereinigte Klassenkriminalitätsrate, nämlich die durchschnittliche Selbstkontrolle der Mitschüler. Auf Seiten der einzelnen Untersuchungsperson tragen alle betrachteten Individualmerkmale signifikant zur Erklärung der Einlassung auf Straftaten bei. Dazu kommt der schon angesprochene direkte Effekt der Peerdelinquenz. Wenn der Anteil der kriminalitätserfahrenen Klassenkameraden um $10 \%$ steigt, wächst die Wahrscheinlichkeit eigener Straffälligkeit um 1,4\%.

Ein systematischer Einfluss der Kriminalität des einzelnen Schülers auf die Delinquenzbelastung der Klassenkameraden konnte hingegen nicht beobachtet werden. Letzteres ist vor dem Hintergrund der Abwesenheit von Möglichkeiten der Mitschülerselektion zu beurteilen. Eine einmal getroffene Klasseneinteilung bleibt in Österreich vier Jahre lang weitgehend stabil - der einzelne Schüler kann nicht bestimmen, mit wem er das Klassenzimmer teilt.

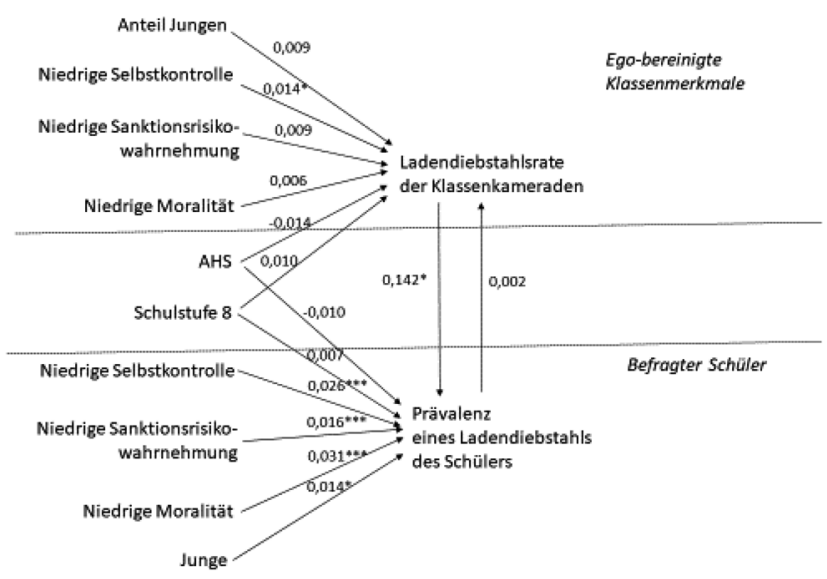

Abbildung 2: Ergebnisse der Schätzung des non-rekursiven Strukturgleichungsmodells

${ }^{\star} \mathrm{p}<0,05 \quad{ }^{\star \star} \mathrm{p}<0,01 \quad{ }^{\star \star \star} \mathrm{p}<0,001 \quad \mathrm{p}=\alpha$-Fehler (einseitig)
Das berechnete Modell zeigt wie schon erwähnt eine exzellente Anpassungsqualität, und auf der individuellen Ebene werden auch mehrere signifikante Bedingungsfaktoren ermittelt. Dies darf aber nicht darüber hinwegtäuschen, dass die Varianzaufklärungsleistung auf der Ebene der Klassenkameraden $\left(R^{2}=0,17\right)$ unbefriedigend ausfällt. Hier wird nur ein signifikanter Effekt, nämlich der der egokorrigierten Selbstkontrolle auf die ego-bereinigte Ladendiebstahlsquote, ausgewiesen. Dieser Befund steht im Einklang mit den Ergebnissen von IVREG2, denen zufolge keine allzu starken Instrumentalvariablen vorliegen. Die in Tabelle 4 berichtete Signifikanz der Steigungskoeffizienten in der vorgeschalteten Regression zur Berechnung der Erwartungswerte der ego-bereinigten Klassendelinquenz ist nur darauf zurückzuführen, dass dort die Clusterstruktur der Daten nicht adäquat berücksichtigt wurde, was auf eine Unterschätzung der Standardfehler und eine Überschätzung der Signifikanzen hinauslief (Bacher 2009). Rechtfertigen lässt sich die Vernachlässigung der geschachtelten Struktur der Daten mit dem Verweis darauf, dass es vormals eben nur um die Ermittlung der Prognosewerte ging. ${ }^{16}$ Wird auch in diesem Schritt der hierarchischen Organisation der Daten Rechnung getragen, was mit IVREG2 möglich ist, ergibt sich ebenfalls nur ein signifikanter Effekt der mangelnden Selbstkontrolle. Wichtig für unsere Zwecke ist in erster Linie der Umstand, dass die berechneten Regressionskoeffizienten (Vorzeichen und Größe) bei beiden Schätzvarianten (gewöhnliche OLS-Regression mit SPSS und GMM-Schätzung mit IVREG2) identisch ausfallen.

\section{Zusammenfassung}

Anliegen der vorliegenden Arbeit war es, den Fragen nachzugehen, ob die aggregierte Delinquenzbelastung einer Schulklasse als Maßzahl für die Testung kriminogener Peer-Effekte taugt, welche Probleme mit der Verwendung dieser Messgröße verbunden sind und wie der Einfluss der Mitschüler auf das eigene Legalverhalten möglichst unverzerrt bestimmt werden kann. In der (deutschsprachigen) kriminologischen Forschung wird die Bedeutung delinquenter Peerexposition für die Kriminalitätshäufigkeit junger Menschen gerne gestützt auf Schülerbefragungen geprüft, indem ein Mehrebenenmodell gerechnet wird, in welchem die Kriminalitätsprävalenzrate der Schulklasse, die der Jugendliche besucht, prädiktorenseitig als Kon-

16 IBM-SPSS ermöglicht leider keine Berechnung clusterungsrobuster Standardfehler. 
textmerkmal einbezogen wird (Hirtenlehner \& Bacher 2017; Weiss 2019; Windzio 2013). Selbst wenn man die Annahme stehen lässt, dass sich die Freunde aus den Klassenkameraden rekrutieren, ist ein solches Vorgehen als zweifelhaft zu betrachten. Zum einen liegt ein Ego-Bias vor, da der Jugendliche, dessen Verhalten man erklären will, zugleich in die Bildung der Expositionsvariablen miteinfließt und dadurch die Messung der Regressoren kontaminiert. Zum zweiten besteht ein Simultanitäts- bzw. Reziprozitätsproblem, da der betreffende Jugendliche nicht nur selbst von seinen Mitschülern beeinflusst wird, sondern dieser mit seinem eigenen Handeln auch auf das Legalverhalten der Klassenkameraden einzuwirken vermag. Das Abstellen auf die hochaggregierte Klassenkriminalitätsrate als Kontextebenenprädiktor wird daher regelmäßig zu einer Überschätzung der realen Peereinflüsse führen. Es kann - wie anhand eines fiktiven Beispiels gezeigt wurde - sogar ein substanzieller Peer-Effekt errechnet werden, wenn ein solcher in den Daten gar nicht vorhanden ist.

Eine statistisch korrekte Bestimmung des Effektes delinquenter Peerexposition kann nur erreicht werden, indem (1.) anstelle der einfach auf Klassenebene hochaggregierten Kriminalitätsprävalenzrate ein ego-korrigierter Messwert verwendet wird, in den nur die Klassenkameraden einfließen, und (2.) bei der Schätzung auch der reziproken Wirkungsdynamik zwischen Schülern und ihren Klassenkameraden Rechnung getragen wird. Letzteres impliziert, dass für die ego-bereinigte Klassenkriminalität Instrumentenvariablen spezifiziert werden müssen, die bestimmte Bedingungen erfüllen. Nach erfolgter Selektion solcher Instrumentenvariablen stehen zwei Analysestrategien zur Verfügung - ein schrittweises Vorgehen im Rahmen der etablierten Instrumentalvariablenregression (Bushway \& Apel 2010) und eine Simultanschätzung der reziproken Wirkungspfade im Bezugsrahmen der Strukturgleichungsmodellierung (Williams 2015). Die beiden Ansätze unterscheiden sich darin, dass bei der Instrumentenvariablenregression nur der (um die Rückkoppelungswirkung korrigierte) Einfluss der Kriminalität der Klassenkameraden auf die Delinquenz des Schülers berechnet wird, während bei der Strukturgleichungsmodellierung beide Effekte - jener der Klassenkameraden auf den Schüler und umgekehrt jener des Schülers auf die Klassenkameraden - zugleich geschätzt werden.

Für das untersuchte Anwendungsbeispiel konnte bei korrekter Spezifikation der vorhandenen Datenkonstellation ein statistisch signifikanter kriminogener Peer-Effekt wiederholt nachgewiesen werden. Sowohl Instrumentalvariablenanalysen als auch nicht-rekursive Strukturgleichungsmodelle geben $\mathrm{zu}$ erkennen, dass die eigene Be- reitschaft zum Entwenden von Waren aus Geschäften mit dem Anteil ladendiebstahlserfahrener Klassenkameraden wächst. Dieser Befund passt zur Beobachtung, dass junge Menschen ihre Ladendiebstahlshandlungen häufig im Beisein gleichaltriger Peers begehen (Farrington 1999).

Die Höhe der ermittelten Regressionskoeffizienten ist als moderat zu beurteilen, was einmal mehr darauf hindeutet, dass korrekte direkte Messungen der Kriminalität im Gleichaltrigenumfeld niedrigere Effekte auf das persönliche Legalverhalten produzieren als Maßzahlen der perzipierten Peerdelinquenz (Hoeben et al. 2016). Der geringe eigene Einfluss auf das Ausmaß der Mitschülerkriminalität ist u. a. dem Umstand geschuldet, dass Kinder und Jugendliche nicht steuern können, wer ihrem Klassenverband angehört. Insofern können Diebstahlsaktivitäten einer Person schulintern kaum Selektionseffekte entfalten.

Obwohl die hier vorgestellten Analysetechniken eine statistisch korrekte Schätzung des von der Gesamtheit der Klassenkameraden ausgehenden kriminogenen Effektes ermöglichen, legen die Ausführungen und Ergebnisse nahe, in Zukunft auch dem Problem der Messung des Umfangs delinquenter Peerexposition mehr Aufmerksamkeit zu widmen. Dem dargestellten Vorgehen liegt die Annahme zugrunde, dass sich die Freunde eines Jugendlichen aus der eigenen Schulklasse rekrutieren. Das muss nicht immer der Fall sein, etwa dann, wenn die Schüler einpendeln und dabei aus unterschiedlichen Städten oder Gemeinden kommen. Klassenkameraden werden daher nur einen begrenzten Ausschnitt des gesamten Freundschaftsnetzwerkes junger Menschen repräsentieren (Friemel \& Knecht 2009).

Gravierender wirkt vermutlich die Tatsache, dass nicht alle Schüler einer Klasse miteinander befreundet sind (und folglich auch Freizeit gemeinsam verbringen), sondern die Freunde nur eine Teilgruppe des Klassenverbandes bilden. In die Berechnung der ego-bereinigten Klassenkriminalitätsrate können zahlreiche Individuen einfließen, die als Bezugsperson und Orientierungsgröße eines Schülers wenig relevant sind. Die Kriminalitätsbelastung der Klassenkameraden wird daher nur ein unscharfes, möglicherweise systematisch verzerrtes Bild von der Delinquenz im Freundeskreis junger Menschen zeichnen. Peer-Effekte sollten dadurch abgeschwächt werden. Etwas abgemildert werden kann dieser Bias, indem man Klassenzimmerbefragungen mit ego-zentrierten Netzwerkanalysen kombiniert, wie Gerstner \& Oberwittler (2015) es beispielsweise tun. Das Identifizieren der tatsächlichen Freunde unter den Klassenkameraden und die Berechnung der Kriminalitätsprävalenzrate nur auf Basis der als Freunde genannten Mitschüler wird eine zielgenauere Messung delinquenter Peerexposition hervorbringen. Es- 
sentiell bleibt dennoch, schon bei der Konstruktion der Erhebungsinstrumente auf die Miteinbeziehung geeigneter Instrumentenvariablen zu achten. In der vorliegenden Anwendung ist das leider nur teilweise gelungen.

\section{Anhang}

\section{IBM-SPSS 24}

Nachfolgende Syntax nimmt zunächst eine EM-Schätzung der fehlenden Werte vor, berechnet anschließend die egokorrigierten Klassenmittelwerte und führt den ersten Schritt der »Two-Stage-Least-Squares«-Schätzung durch. Abschließend werden die Datenfiles für Mplus und STATA erzeugt und abgespeichert.

cd »C: \bacher \ladendiebstahl \data $\backslash \ll$.

GET FILE='Bacher.sav' .

DATASET NAME DataSet 1 WINDOW=FRONT .

recode schulform $(1=1)(0=0)$ into ahs.

recode schulstufe $(1=1)(0=0)$ into stufe 8 .

recode $\operatorname{sex}(0=0)(1=1)$ into junge.

crosstab tab=schulform by ahs/schulstufe by stufe8/ sex by junge.

MVA VARIABLES=classidflnr sat_morality sat_selfcont sat deter junge ahs

stufe8 ld12jastadt

/EM sat_morality sat_selfcont sat_deter junge ahs stufe8 stadt (TOLERANCE $=0.001$ CONVERGENCE $=0.0001$ ITERATIONS=25 outfile="bacherEM.sav《) .

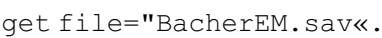

DATASET NAME Dataset 1 WINDOW=FRONT .

*Ego-bereinigte Durchschnittswerte berechnen.

aggregate outfile=* mode=addvariables overwrite=yes

/break classid

$/ \mathrm{xnclass}=\mathrm{nu}$

lxsat_deterxsat_moralityxsat_selfcont $=$

sum (sat_detersat_moralitysat_selfcont)

$/ x j$ unge $=$ sum $(j$ unge $)$

$/ x l d 12 j a=\operatorname{sum}(1 d 12 j a)$

/yyalle=mean $(l d 12 j a)$.

*Klassen mit mehr als 9 Schülern auswählen. selectif (xnclass>9). compute xnclass_i=xnclass -1 .

compute xld12ja_i=(xld12ja-ld12ja)/xnclass_i.

compute xsat_deter_i=(xsat_deter - sat_deter $) / \mathrm{xncl}$ ass_i.

compute xsat_morality_i=(xsat_morality - sat_morali ty)/xnclass_i.

compute xsat_selfcont_i=(xsat_selfcont - sat_selfcont)/xnclass_i.

computexjunge_i=(xjunge $-j$ unge $) /$ xnclass_i.

* Standardisieren der Individualmerkmale zur besseren

*Interpretation der Effekte.

desc var=sat_detersat_moralitysat_selfcont/save.

*Berechnung der Erwartungswerte für Delinquenzrate der Schulkameraden.

delete variables yy.

regression var=xld12ja_i xsat_selfcont_i xsat_de ter_ixsat_morality_ixjunge_i

ahs stufe8 zsat_selfcont zsat_deter zsat_morality junge

/dep=xld12ja_i/method=enter xsat_selfcont_i xsat deter_i

xsat_morality_ixjunge_i ahs stufe8

zsat_selfcontzsat_deter zsat_morality junge

/ save pred (yy).

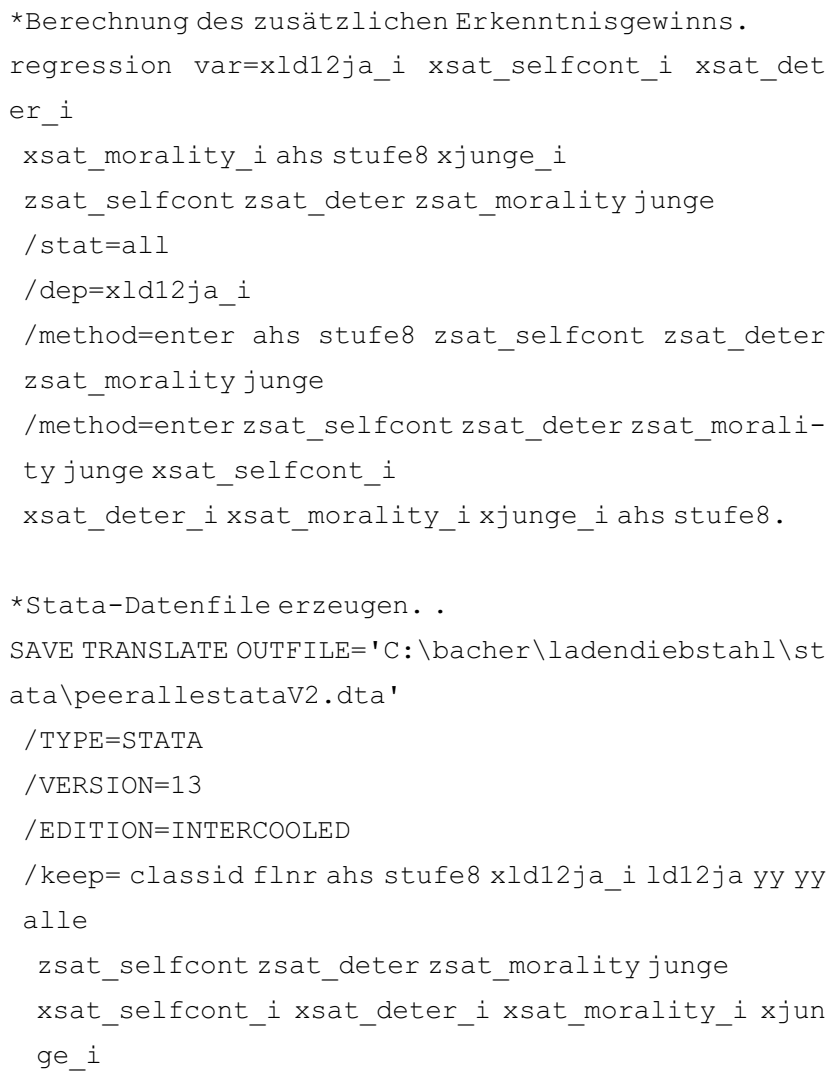




\section{/MAP}

/REPLACE.

SAVE TRANSLATE OUTFILE="C: \bacher $\backslash$ ladendiebstahl

$\backslash$ mplus \peerallev2.dat"

$/ \mathrm{TYPE}=\mathrm{TAB}$

/ textoptions decimal $=\operatorname{dot}$

/keep=classid flnr ahs stufe8 xld12ja xld12ja_i

ld12ja yy yyalle

zsat_selfcont zsat_deter zsat_morality junge

xsat_selfcont_i xsat_deter_i xsat_morality_i xjun

ge_i

$/ \mathrm{MAP}$

/ REPLACE

/CELLS=VALUES.

\section{STATA 14}

Die Befehlssyntax für das Einlesen der Daten und die robuste GMM-Schätzung lautet:

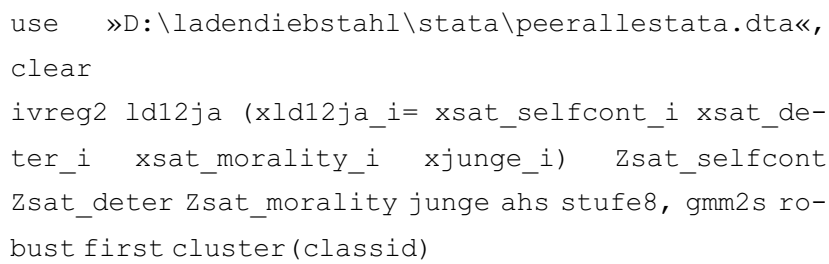

\section{Mplus 8.2}

Der Input-File für die Schätzung des nicht-rekursiven Strukturgleichungsmodells wird dargestellt.

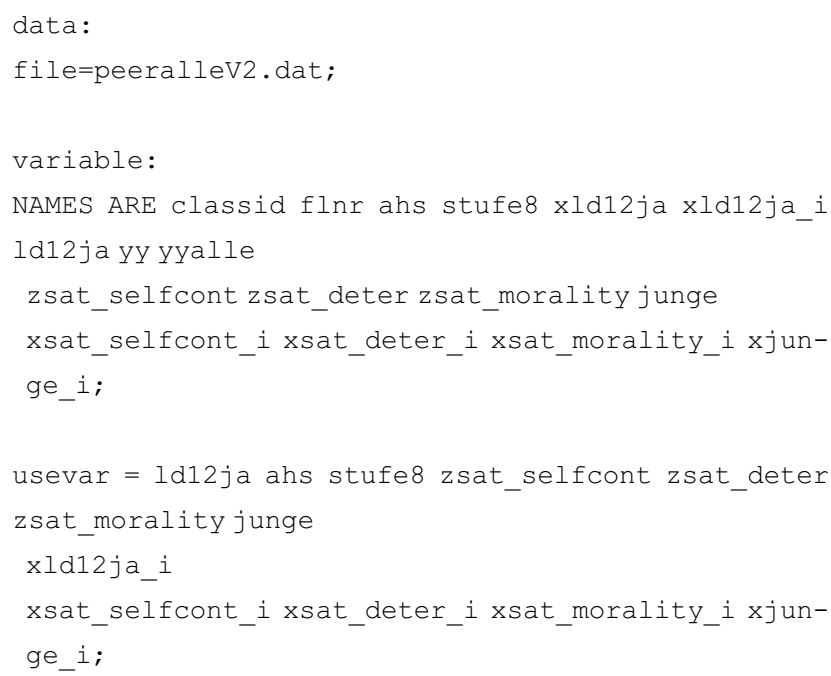

cluster $=$ classid;

missing are all (-99);

define:

ANALYSIS : estimator=mlr;

TYPE IS complex;

MODEL :

ld12ja oN zsat_selfcont zsat_deter zsat_morality junge xld12ja_i ahs stufe8;

xld12ja_i on ahs stufe8 xsat_selfcont_ixsat_deter_i xsat_morality_ixjunge_i;

ld12ja ON xld12ja_i;

xld12ja_i ON ld12ja;

OUTPUT: sampstat TECH2 standardized modindices (1) residual;

\section{Literatur}

Angrist, J.D. (2006). Instrumental variables methods in experimental criminological research: what, why and how. Journal of Experimental Criminology 2 (1), 23-44.

Angrist, J.D. \& Pischke, J.-S. (2009). Mostly Harmless Econometrics. An Empiricist's Companion. Princeton: Princeton University Press.

Bacher, J. (2009). Analyse komplexer Stichproben. In M. Weichbold, J. Bacher \& C. Wolf (Hrsg.), Umfrageforschung. Herausforderungen und Grenzen (253-272). Wiesbaden: VS Verlag für Sozialwissenschaften.

Bascle, G. (2008). Controlling for endogeneity with instrumental variables in strategic management research. Strategic Organization 6 (3), 285-327.

Baum, C., Schaffer, M.E. \& Stillman, S. (2003). Instrumental variables and GMM: Estimation and testing. The Stata Journal 3 (1), 1-31.

Baum, C., Schaffer, M.E. \& Stillman, S. (2007). Enhanced routines for instrumental variables/generalized method of moments estimation and testing. The Stata Journal 7 (1), 465-506.

Bollen, K.A. (2012). Instrumental variables in sociology and the social sciences. Annual Review of Sociology 38 (1), 37-72.

Bushway, S.D. \& Apel, R.J. (2010). Instrumental variables in criminology and criminal justice. In A. Piquero \& D. Weisburd (eds.), Handbook of Quantitative Criminology (595-612). New York: Springer.

Cameron, A.C. \& Trivedi, P.K. (2010). Microeconomics Using Stata. College Station: Sztata Press.

Duncan, O.D., Haller, A.O. \& Portes, A. (1968). Peer influences on aspirations: a reinterpretation. American Journal of Sociology 74 (2), 119-137. 
Farrington, D.P. (1999). Measuring, explaining and preventing shoplifting: A review of British Research. Security Journal 12 (1), 9-27.

Friemel, T. \& Knecht, A. (2009). Praktikable vs. tatsächliche Grenzen von sozialen Netzwerken. Eine Diskussion zur Validität von Schulklassen als komplette Netzwerke. In R. Häußling (Hrsg.), Grenzen von Netzwerken (15-32). Wiesbaden: VS Verlag für Sozialwissenschaften.

Geiser, C. (2011). Datenanalyse mit Mplus. Eine anwendungsorientierte Einführung. Wiesbaden: VS Verlag für Sozialwissenschaften.

Gerstner, D. \& Oberwittler, D. (2015). Wer kennt wen und was geht ab? Ein netzwerkanalytischer Blick auf die Rolle delinquenter Peers im Rahmen der Situational Action Theory. Monatsschrift für Kriminologie und Strafrechtsreform 98 (3), 204-226.

Haynie, D.L. \& Osgood, W.D. (2005). Reconsidering peers and delinquency: How do peers matter? Social Forces 84 (2), 1109-1130.

Hellevik, O. (2009). Linear versus logistic regression when the dependent variable is a dichotomy. Quality \& Quantity 43 (1), 59-74.

Hirtenlehner, H. (2019). Does perceived peer delinquency amplify or mitigate the deterrent effect of perceived sanction risk? Deviant Behavior 40 (3), 361-384.

Hirtenlehner, H. \& Bacher, J. (2017). Interaktive Beziehungsdynamiken am Beispiel der Ladendiebstahlsdelinquenz junger Menschen. Monatsschrift für Kriminologie und Strafrechtsreform 100 (6), 404-429.

Hoeben, E.M., Meldrum, R.C., Walker, D.A. \& Young, J.T. (2016). The role of peer delinquency and unstructured socializing in explaining delinquency and substance use: A state-of-the-art review. Journal of Criminal Justice 47 (1), 108-122.

Hu, L.-T. \& Bentler, P.M. (1999). Cutoff criteria for fit indexes in covariance structure analysis: Conventional criteria versus new alternatives. Structural Equation Modeling: A Multidisciplinary Journal 6 (1), 1-55.

Johnston, J. (1984). Econometric Methods. New York: McGraw-Hill.

Kim, J. \& Fletcher, J.M. (2018). The Influence of classmates on adolescent criminal activities in the United States. Deviant Behavior 39 (3), 275-292.

Lousdal, M.L. (2018). An introduction to instrumental variable assumptions, validation and estimation. Emerging Themes in Epidemiology 15 (1), 1-7.

Matsueda, R.L. \& Anderson, K. (1998). The dynamics of delinquent peers and delinquent behavior. Criminology 36 (2), 269-308.

Muthén, L.K. \& Muthén, B.O. (2017). Mplus User's Guide. $8^{\text {th }}$ ed. Los Angeles: Muthén \& Muthén.
Oberwittler, D. (2004). Stadtstruktur, Freundeskreise und Delinquenz: Eine Mehrebenenanalyse zu sozialökologischen Kontexteffekten auf schwere Jugenddelinquenz. In D. Oberwittler \& S. Karstedt (Hrsg.), Soziologie der Kriminalität (135-170). Wiesbaden: Springer VS.

Pratt, T.C., Cullen, F.T., Sellers, C.S., Winfree, L.T., Madensen, T.D., Daigle, L.E. et al. (2010). The empirical status of social learning theory: A meta-analysis. Justice Quarterly 27 (6), 765-802.

Rebellon, C.J. \& Modecki, K.L. (2014). Accounting for projection bias in models of delinquent peer influence: The utility and limits of latent variable approaches. Journal of Quantitative Criminology 30 (2), 163-186.

Reinecke, J. (2014). Strukturgleichungsmodelle in den Sozialwissenschaften. München: De Gruyter Oldenbourg.

Reisinger, M., Svecnik, E. \& Schwetz, H. (2012). Fehlende Werte und keine Normalverteilung? Tipps und Abhilfen für das quantitativ orientierte Forschen. Wien: Facultas.

Snijder, T. \& Bosker, R. (2004). Multilevel Analysis. An Introduction to Basic and Advanced Multilevel Modeling. London: Sage.

Staiger, D. \& Stock, J.H. (1997). Instrumental variables regression with weak instruments. Econometrica 65 (3), 557-586.

Stock, J.H. \& Trebbi, F. (2003). Who invented instrumental variable regression? Journal of Economic Perspectives 17 (3), 177-194.

Stock, J.H. \& Yogo, M. (2005). Testing for weak instruments in linear IV regression. In D.W. Andrews (ed.), Identification and Inference for Econometric Models (80-108). Cambridge: Cambridge University Press.

Svensson, R. \& Oberwittler, D. (2010). It's not the time they spend, it's what they do: The interaction between delinquent friends and unstructured routine activity on delinquency. Journal of Criminal Justice 38 (5), 1006-1014.

Warr, M. (2002). Companions in Crime. The Social Aspects of Criminal Conduct. Cambridge: Cambridge University Press.

Weiss, M. (2019). Einfluss des Klassenkontextes auf die Delinquenzentwicklung. In S. Wallner, M. Weiss, J. Reinecke \& M. Stemmler (Hrsg.), Devianz und Delinquenz in Kindheit und Jugend (187203). Wiesbaden: Springer VS.

Williams, R. (2015). Nonrecursive Models (Extended Version). Indiana: University of Notre Dame; https://www3.nd.edu/ rwilliam/ stats2/193x.pdf [20.04.2019].

Windzio, M. (2013). Räumliche Diffusion expressiver Delinquenz in Schulen und Stadtbezirken. In D. Oberwittler, S. Rabold \& D. Baier (Hrsg.), Städtische Armutsquartiere - Kriminelle Lebenswelten? (193-216). Wiesbaden: Springer VS. 